\title{
Clinically nonfunctioning pituitary adenoma growth after radiosurgery
}

\section{Crescimento de adenoma pituitário clinicamente não funcionante após radiocirurgia}

Rafael Loch Batista, Andrea Cecilia Toscanini, Andrea Glezer, Mário Gilberto Siqueira, Salomon Benabou, Erick Talamoni Fonoff, Wagner Malagó Tavares, Manoel Jacobsen Teixeira, Malebranche Berardo Carneiro da Cunha Neto

Departamento de Neurocirurgia Funcional da Divisão de Neurocirurgia Funcional do Hospital das Clínicas da Universidade de São Paulo (USP), São Paulo SP, Brazil. Correspondence: Rafael Loch Batista; Hospital das Clínicas da Universidade de São Paulo; Alameda Joaquim Eugênio de Lima 1.058/102;01403-002 São Paulo SP Brasil; E-mail: r-loch@uol.com.br

Conflict of interest: There is no conflict of interest to declare.

Received 29 February 2012; Received in final form 26 March 2012; Accepted 02 April 2012

Radiosurgery (RS) is a minimally invasive technique suitable for lesions of the central nervous system (CNS), with $<3 \mathrm{~cm}$ in diameter or volume $<30 \mathrm{~mL}$. Benign tumors of the CNS are candidates to perform $\mathrm{RS}^{1}$. It has emerged as a therapeutic option for clinically nonfunctioning pituitary adenomas (CNFPA), associated with effective control of tumor growth and few complications ${ }^{1}$. About $30 \%$ of pituitary adenomas are classified as CNFPA, and patients with this tumor usually have clinical symptoms, such as headaches, visual loss, hypopituitarism and, less commonly, pituitary apoplexy ${ }^{2}$. These tumors often develop slowly, but diagnosis tends to be late. Surgery is the primary treatment option, preferably transsphenoidal, complemented or not by radiotherapy (RT) or RS ${ }^{1}$. In cases of tumor residues, the therapeutic approach remains controversial.

Efficacy and safety of RS in CNFPA have not been evaluated as adequately as in functioning pituitary adenomas ${ }^{1}$. In three series of patients, with a follow-up period longer

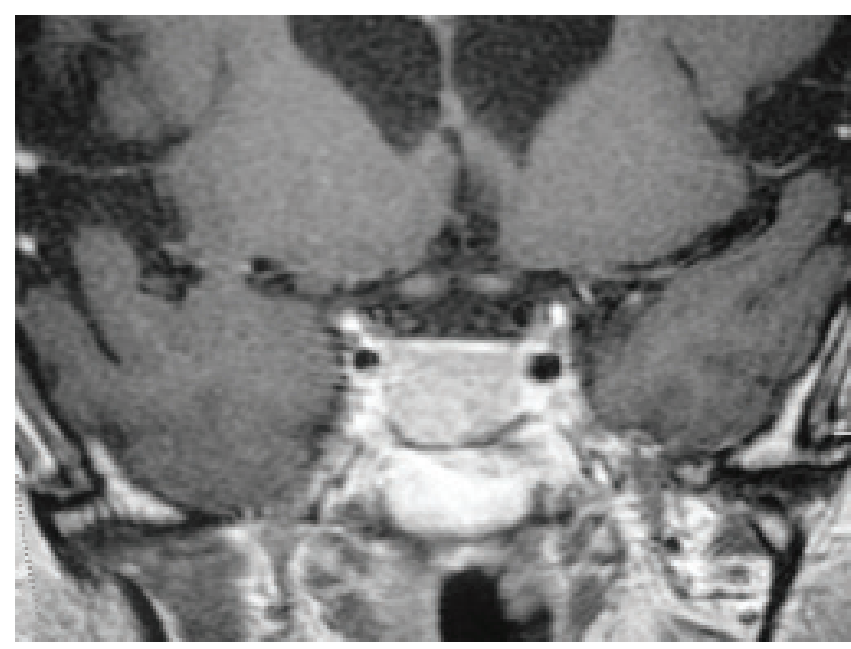

Fig 1. Before radiosurgery. Cranial nuclear magnetic resonance scan from March, 2004, revealing probable tumor residues, measuring $2.5 \times 2.0 \times 1.5 \mathrm{~cm}$, with greater involvement of the sphenoid sinus. 

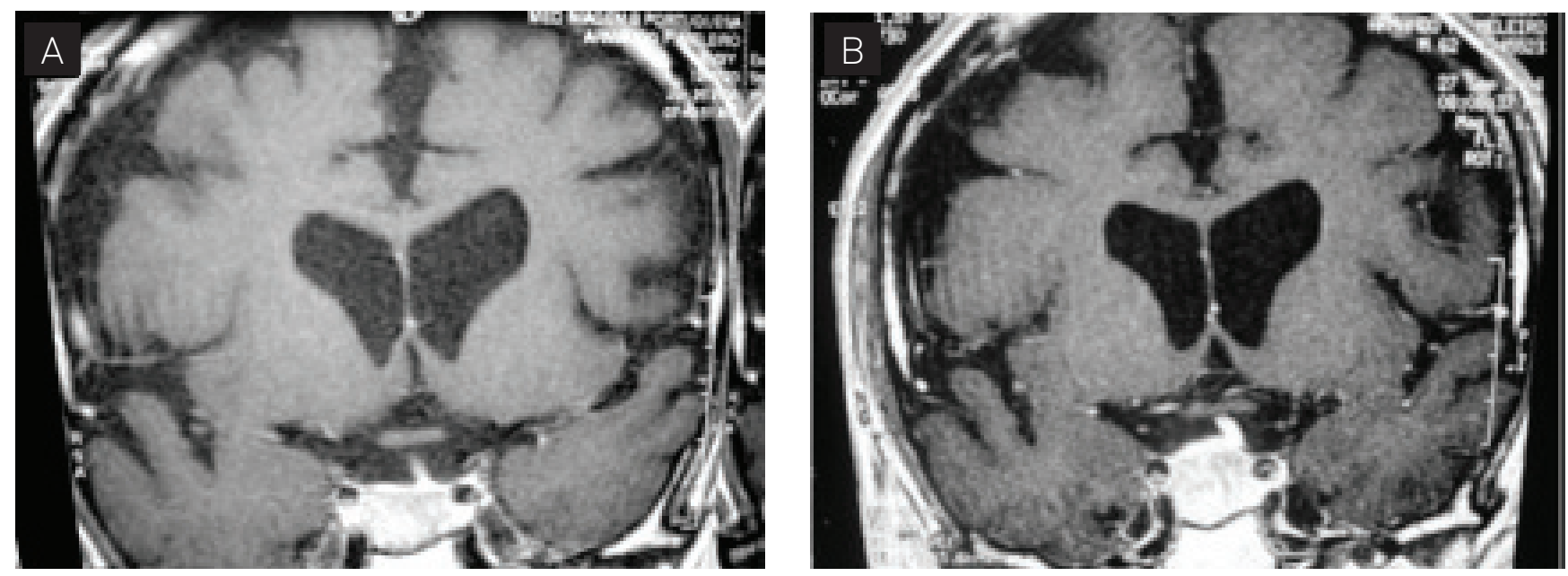

Fig 2. After radiosurgery. (A) Image from June, 2004 (three months after radiosurgery); (B) image from September, 2004 (six months after radiosurgery), both showing growth of the sellar lesion.

than 48 months, RS was able to control tumor growth in 93 to $96 \%$ of the cases $^{1-3}$.

Complications of RS are optic neuropathy, lesions in adjacent vascular structures, parenchymal brain injury, hypopituitarism, and neoplasms ${ }^{1-4}$. Stenosis or occlusion of the internal carotid artery has already been reported ${ }^{5}$.

\section{CASE}

We reported a case of a patient with CNFPA who underwent RS and showed tumor growth after it.

A 59-year-old male patient, single, born in São Paulo, Brazil, reported that a cranial computed tomography (CCT) performed in 1992 by headache had identified sellar and suprasellar tumors with $2.0 \times 2.0 \mathrm{~cm}$. In 2001, the CCT was repeated and revealed a slight increase $(2.2 \times 2.0 \mathrm{~cm})$ of them. The patient denied headaches or visual loss.

Hormonal evaluation did not identify any changes. Nuclear magnetic resonance (NMR) scan showed sellar tumor with supra- and infrasellar extension, measuring $2.2 \mathrm{x}$ $2.5 \times 2.8 \mathrm{~cm}$, isointense on $\mathrm{T} 1$ and hyperintense on $\mathrm{T} 2$, with compression of the optic chiasm. Transsphenoidal pituitary surgery was performed in 2001.
The anatomicopathologic diagnosed pituitary adenoma with discrete anaplasia and sites reagent for thyroid-stimulating hormone (TSH) in the cytoplasm.

Cranial NMR was repeated in 2001, 2002, and 2003, and scans revealed intrasellar content, with no signs indicating tumor residues. In 2004, NMR demonstrated residues with $2.5 \times 2.0 \times 1.5 \mathrm{~cm}$.

RS was underwent in March, 2004 (Fig 1). NMR sellar was underwent in June and September, 2004 (Fig 2), with lesion growth in six months. In January, 2005, NMR revealed a significant reduction in tumor volume to about $1.5 \mathrm{~cm}$.

A possible explanation to growth and subsequently regression after RS is the occurrence of apoplexy. Reports of pituitary apoplexy cases after RT have promoted a relationship between apoplexy and radiation ${ }^{1}$. Radiation is known to increase vascularization of pituitary adenomas, therefore leading to apoplexy.

RS is an alternative for the treatment of CNFPA, however, there are no reports of pituitary apoplexy or tumor growth after RS. A possible reabsorption of the apoplectic content leading to subsequent regression is likely. Tumor growth after RS, probably due to pituitary apoplexy, may be a possible complication of this therapeutic modality.

\section{References}

1. Laws ER, Sheehan JP, Sheehan JM, Jagnathan J, Jane JA, Oskouian R. Stereotatic radiosurgery for pituitary adenomas: a review of the literature.J Neur-Oncol 2004;69:257-272.

2. Sanno N, Teramoto A, Osamura RY, et al. Pathology of pituitary tumours. Neurosurg Clin N Am 2003;14:25-39.

3. Laws ER, Vance ML. Radiosurgery for pituitary tumours and craniopharyngiomas. Neurosurg Clin North Am 1999;10:327-336.
4. Akabane A, Yamada S, Jokura H. Gamma knife radiosurgery for pituitary adenomas. Endocrine 2005;28:87-92.

5. Muramatsu J, Yoshida M, Shioura H, et al. Clinical results of LINACbased stereotactic radiosurgery for pituitary adenoma. Nippon Igaku Hoshasen Gakkai Zasshi 2003;63:225-230. 